Internat. J. Math. \& Math. Sci.

Vol. 23, No. 10 (2000) 703-709

S0161171200002738

(C) Hindawi Publishing Corp.

\title{
LOCAL PROPERTIES OF FOURIER SERIES
}

\section{HÜSEYIN BOR}

\author{
(Received 11 February 1999)
}

\begin{abstract}
A theorem on local property of $\left|\bar{N}, p_{n}\right|_{k}$ summability of factored Fourier series, which generalizes some known results, and also a general theorem concerning the $\left|\bar{N}, p_{n}\right|_{k}$ summability factors of Fourier series have been proved.
\end{abstract}

Keywords and phrases. Absolute summability, Fourier series, local property.

2000 Mathematics Subject Classification. Primary 40D15, 40G99, 42A24, 42B15.

1. Introduction. Let $\sum a_{n}$ be a given infinite series with partial sums $\left(s_{n}\right)$. Let $\left(p_{n}\right)$ be a sequence of positive numbers such that

$$
P_{n}=\sum_{v=0}^{n} p_{v} \rightarrow \infty \quad \text { as } n \longrightarrow \infty,\left(P_{-i}=p_{-i}=0, i \geq 1\right) .
$$

The sequence-to-sequence transformation

$$
t_{n}=\frac{1}{P_{n}} \sum_{v=0}^{n} p_{v} s_{v}
$$

defines the sequence $\left(t_{n}\right)$ of the $\left(\bar{N}, p_{n}\right)$ mean of the sequence $\left(s_{n}\right)$ generated by the sequence of coefficients $\left(p_{n}\right)$ (see [8]).

The series $\sum a_{n}$ is said to be summable $\left|\bar{N}, p_{n}\right|_{k}, k \geq 1$, if (see [4])

$$
\sum_{n=1}^{\infty}\left(\frac{P_{n}}{p_{n}}\right)^{k-1}\left|t_{n}-t_{n-1}\right|^{k}<\infty .
$$

In the special case when $p_{n}=1$ for all values of $n$ (resp., $k=1$ ), $\left|\bar{N}, p_{n}\right|_{k}$ summability is the same as $|C, 1|$ (resp., $\left.\left|\bar{N}, p_{n}\right|\right)$ summability. Also if we take $k=1$ and $p_{n}=1 / n$ summability $\left|\bar{N}, p_{n}\right|_{k}$, is equivalent to the summability $|R, \log n, 1|$. A sequence $\left(\lambda_{n}\right)$ is said to be convex if $\Delta^{2} \lambda_{n} \geq 0$ for every positive integer $n$, where $\Delta^{2} \lambda_{n}=\Delta \lambda_{n}-\Delta \lambda_{n+1}$ and $\Delta \lambda_{n}=\lambda_{n}-\lambda_{n+1}$.

Let $f(t)$ be a periodic function with period $2 \pi$, and integrable $(L)$ over $(-\pi, \pi)$. Without loss of generality we may assume that the constant term in the Fourier series of $f(t)$ is zero, so that

$$
\int_{-\pi}^{\pi} f(t) d t=0
$$

and

$$
f(t) \sim \sum_{n=1}^{\infty}\left(a_{n} \cos n t+b_{n} \sin n t\right)=\sum_{n=1}^{\infty} A_{n}(t)
$$


It is familiar that the convergence of the Fourier series at $t=x$ is a local property of $f$ (i.e., it depends only on the behaviour of $f$ in an arbitrarily small neighbourhood of $x$ ), and hence the summability of the Fourier series at $t=x$ by any regular linear summability method is also a local property of $f$. The local property problem of the factored Fourier series have been studied by several authors (see [1, 2, 5, 6, 7, 9]). Few of them are given below.

2. Mohanty [13] has demonstrated that the $|R, \log n, 1|$ summability of the factored Fourier series

$$
\sum \frac{A_{n}(t)}{\log (n+1)}
$$

at $t=x$, is a local property of the generating function of $f$, whereas the $|C, 1|$ summability of this series is not. Later on, Matsumoto [10] improved this result by replacing the series (2.1) by

$$
\sum \frac{A_{n}(t)}{[\log \log (n+1)]^{\delta}}, \quad \delta>1 .
$$

Generalizing the above result Bhatt [3] proved the following theorem.

THEOREM 2.1. If $\left(\lambda_{n}\right)$ is a convex sequence such that $\sum n^{-1} \lambda_{n}$ is convergent, then the summability $|R, \log n, 1|$ of the series $\sum A_{n}(t) \lambda_{n} \log n$ at a point can be ensured by a local property.

Mishra [12] has proved the following theorem by replacing the factor $\left(\lambda_{n} \log n\right)$ in the most general form.

THEOREM 2.2. Let the sequence $\left(p_{n}\right)$ be such that

$$
P_{n}=O\left(n p_{n}\right), \quad P_{n} \Delta p_{n}=O\left(p_{n} p_{n+1}\right) .
$$

Then the summability $\left|\bar{N}, p_{n}\right|$ of the series

$$
\sum_{n=1}^{\infty} \frac{A_{n}(t) \lambda_{n} P_{n}}{n p_{n}},
$$

at a point can be ensured by local property, where $\left(\lambda_{n}\right)$ is as in Theorem 2.1.

But this theorem does not directly generalize any of the above mentioned results involving $|R, \log n, 1|$ summability since order relations are not satisfied by $p_{n}=1 / n$.

3. The aim of this paper is to prove a more general theorem which includes some of the above mentioned results as special cases.

Now, we shall prove the following theorem.

THEOREM 3.1. Let $k \geq 1$. If $\left(\lambda_{n}\right)$ is a convex sequence such that $\sum p_{n} \lambda_{n}$ is convergent, then the summability $\left|\bar{N}, p_{n}\right|_{k}$ of the series $\sum A_{n}(t) \lambda_{n} P_{n}$ at a point can be ensured by a local property.

We need the following lemmas for the proof of our theorem. 
LEMMA 3.2 [11]. If $\left(\lambda_{n}\right)$ is a convex sequence such that $\sum p_{n} \lambda_{n}$ is convergent, where $\left(p_{n}\right)$ is a sequence of positive numbers such that $P_{n} \rightarrow \infty$ as $n \rightarrow \infty$, then $\left(\lambda_{n}\right)$ is a nonnegative monotonic decreasing sequence tending to zero, $P_{n} \lambda_{n}=o$ (1) as $n \rightarrow \infty$ and $\sum P_{n} \Delta \lambda_{n}<\infty$.

LEMMA 3.3. Let $k \geq 1$. If $\left(\lambda_{n}\right)$ is a convex sequence such that $\sum p_{n} \lambda_{n}$ is convergent and $\left(s_{n}\right)$ is bounded, then the series $\sum a_{n} \lambda_{n} P_{n}$ is summable $\left|\bar{N}, p_{n}\right|_{k}$.

Proof. Let $\left(T_{n}\right)$ be the sequence of $\left(\bar{N}, p_{n}\right)$ mean of the series $\sum a_{n} \lambda_{n} P_{n}$. Then, by definition, we have

$$
T_{n}=\frac{1}{P_{n}} \sum_{v=0}^{n} p_{v} \sum_{r=0}^{v} a_{r} \lambda_{r} P_{r}=\frac{1}{P_{n}} \sum_{v=0}^{n}\left(P_{n}-P_{v-1}\right) a_{v} \lambda_{v} P_{v}
$$

Then, for $n \geq 1$, we have

$$
T_{n}-T_{n-1}=\frac{p_{n}}{P_{n} P_{n-1}} \sum_{v=1}^{n} P_{v-1} P_{v} a_{v} \lambda_{v}
$$

By Abel's transformation, we have

$$
\begin{aligned}
T_{n}-T_{n-1}= & \frac{p_{n}}{P_{n} P_{n-1}} \sum_{v=1}^{n-1} P_{v} P_{v} s_{v} \Delta \lambda_{v}-\frac{p_{n}}{P_{n} P_{n-1}} \sum_{v=1}^{n-1} P_{v} s_{v} p_{v} \lambda_{v} \\
& -\frac{p_{n}}{P_{n} P_{n-1}} \sum_{v=1}^{n-1} P_{v} p_{v+1} s_{v} \lambda_{v+1}+s_{n} p_{n} \lambda_{n} \\
= & T_{n, 1}+T_{n, 2}+T_{n, 3}+T_{n, 4} .
\end{aligned}
$$

By Minkowski's inequality for $k>1$, to complete the proof of Lemma 3.3, it is sufficient to show that

$$
\sum_{n=1}^{\infty}\left(\frac{P_{n}}{p_{n}}\right)^{k-1}\left|T_{n, r}\right|^{k}<\infty, \quad \text { for } r=1,2,3,4 .
$$

Now, applying Hölder's inequality with indices $k$ and $k^{\prime}$, where $1 / k+1 / k^{\prime}=1$, we get that

$$
\sum_{n=2}^{m+1}\left(\frac{P_{n}}{p_{n}}\right)^{k-1}\left|T_{n, 1}\right|^{k} \leq \sum_{n=2}^{m+1} \frac{p_{n}}{P_{n} P_{n-1}}\left\{\sum_{v=1}^{n-1}\left|s_{v}\right|^{k} P_{v} P_{v} \Delta \lambda_{v}\right\} \times\left\{\frac{1}{P_{n-1}} \sum_{v=1}^{n-1} P_{v} P_{v} \Delta \lambda_{v}\right\}^{k-1} .
$$

Since

$$
\sum_{v=1}^{n-1} P_{v} P_{v} \Delta \lambda_{v} \leq P_{n-1} \sum_{v=1}^{n-1} P_{v} \Delta \lambda_{v}
$$

it follows by Lemma 3.2 that

$$
\frac{1}{P_{n-1}} \sum_{v=1}^{n-1} P_{v} P_{v} \Delta \lambda_{v} \leq \sum_{v=1}^{n-1} P_{v} \Delta \lambda_{v}=O(1) \quad \text { as } m \longrightarrow \infty .
$$


Therefore

$$
\begin{aligned}
\sum_{n=2}^{m+1}\left(\frac{P_{n}}{p_{n}}\right)^{k-1}\left|T_{n, 1}\right|^{k} & =O(1) \sum_{n=2}^{m+1} \frac{p_{n}}{P_{n} P_{n-1}} \sum_{v=1}^{n-1}\left|s_{v}\right|^{k} P_{v} P_{v} \Delta \lambda_{v} \\
& =O(1) \sum_{v=1}^{m}\left|s_{v}\right|^{k} P_{v} P_{v} \Delta \lambda_{v} \sum_{n=v+1}^{m+1} \frac{p_{n}}{P_{n} P_{n-1}} \\
& =O(1) \sum_{v=1}^{m} P_{v} \Delta \lambda_{v}=O(1) \quad \text { as } m \rightarrow \infty,
\end{aligned}
$$

by virtue of the hypotheses and Lemma 3.2. Again

$$
\begin{aligned}
\sum_{n=2}^{m+1}\left(\frac{P_{n}}{p_{n}}\right)^{k-1}\left|T_{n, 2}\right|^{k} & \leq \sum_{n=2}^{m+1} \frac{p_{n}}{P_{n} P_{n-1}}\left\{\sum_{v=1}^{n-1}\left|s_{v}\right|^{k}\left(P_{v} \lambda_{v}\right)^{k} p_{v}\right\} \times\left\{\frac{1}{P_{n-1}} \sum_{v=1}^{n-1} p_{v}\right\}^{k-1} . \\
& =O(1) \sum_{v=2}^{m+1} \frac{p_{n}}{P_{n} P_{n-1}} \sum_{v=1}^{n-1}\left|s_{v}\right|^{k}\left(P_{v} \lambda_{v}\right)^{k} p_{v} \\
& =O(1) \sum_{v=1}^{m}\left|s_{v}\right|^{k}\left(P_{v} \lambda_{v}\right)^{k} p_{v} \sum_{n=v+1}^{m+1} \frac{p_{n}}{P_{n} P_{n-1}} \\
& =O(1) \sum_{v=1}^{m}\left|s_{v}\right|^{k}\left(P_{v} \lambda_{v}\right)^{k} \frac{p_{v}}{P_{v}} \\
& =O(1) \sum_{v=1}^{m}\left|s_{v}\right|^{k}\left(P_{v} \lambda_{v}\right)^{k-1} p_{v} \lambda_{v} \\
& =O(1) \sum_{v=1}^{m} p_{v} \lambda_{v}=O(1) \quad \text { as } m \rightarrow \infty
\end{aligned}
$$

by virtue of the hypotheses and Lemma 3.2. Using the fact that $P_{v}<P_{v+1}$, similarly we have that

$$
\sum_{n=2}^{m+1}\left(\frac{P_{n}}{p_{n}}\right)^{k-1}\left|T_{n, 3}\right|^{k}=O(1) \sum_{v=1}^{m} p_{v+1} \lambda_{v+1}=O(1) \quad \text { as } m \longrightarrow \infty,
$$

Finally, we have that

$$
\begin{aligned}
\sum_{n=1}^{m}\left(\frac{P_{n}}{p_{n}}\right)^{k-1}\left|T_{n, 4}\right|^{k} & =\sum_{n=1}^{m}\left|s_{n}\right|^{k}\left(P_{n} \lambda_{n}\right)^{k-1} p_{n} \lambda_{n} \\
& =O(1) \sum_{n=1}^{m} p_{n} \lambda_{n}=O(1) \quad \text { as } m \rightarrow \infty,
\end{aligned}
$$

by virtue of the hypotheses and Lemma 3.2. Therefore, we get that

$$
\sum_{n=1}^{m}\left(\frac{P_{n}}{p_{n}}\right)^{k-1}\left|T_{n, r}\right|^{k}=O(1) \quad \text { as } m \rightarrow \infty, \text { for } r=1,2,3,4 .
$$

This completes the proof of Lemma 3.3. 
In the particular case if we take $p_{n}=1$ for all values of $n$ in this lemma, then we get the following corollary.

COROLLARY 3.4. Let $k \geq 1$. If $\left(\lambda_{n}\right)$ is a convex sequence such that $\sum \lambda_{n}$ is convergent and $\left(s_{n}\right)$ is bounded, then the series $\sum n a_{n} \lambda_{n}$ is summable $|C, 1|_{k}$.

Proof of TheOrem 3.1. Since the behaviour of the Fourier series, as far as convergence is concerned, for a particular value of $x$ depends on the behaviour of the function in the immediate neighbourhood of this point only, hence the truth of the theorem is a necessary consequence of Lemma 3.3. If we take $p_{n}=1$ for all values of $n$ in this theorem, then we get a local property result concerning the $|C, 1|_{k}$ summability.

Now we shall prove the following theorem for $\left|\bar{N}, p_{n}\right|_{k}$ summability factors of Fourier series.

THEOREM 3.5. Let $k \geq 1$ and let $\left(\lambda_{n}\right)$ be a convex sequence such that $\sum p_{n} \lambda_{n}<\infty$, where $\left(p_{n}\right)$ is a sequence of positive numbers such that $P_{n} \rightarrow \infty$. If $\sum_{v=1}^{n} P_{v} A_{v}(t)=$ $O\left(P_{n}\right)$, then the series $\sum A_{n}(t) P_{n} \lambda_{n}$ is summable $\left|\bar{N}, p_{n}\right|_{k}$, where $A_{v}(t)$ is as in (1.5).

Proof. Let $T_{n}(t)$ denotes the $\left(\bar{N}, p_{n}\right)$ mean of the series $\sum A_{n}(t) P_{n} \lambda_{n}$. Then, by definition, we have

$$
T_{n}=\frac{1}{P_{n}} \sum_{v=0}^{n} p_{v} \sum_{r=0}^{v} A_{r}(t) P_{r} \lambda_{r}=\frac{1}{P_{n}} \sum_{v=0}^{n}\left(P_{n}-P_{v-1}\right) A_{v}(t) \lambda_{v} P_{v} .
$$

Then, for $n \geq 1$, we have

$$
T_{n}(t)-T_{n-1}(t)=\frac{p_{n}}{P_{n} P_{n-1}} \sum_{v=1}^{n} P_{v-1} P_{v} A_{v}(t) \lambda_{v} .
$$

By Abel's transformation, we have

$$
\begin{aligned}
T_{n}(t)-T_{n-1}(t) & =\frac{p_{n}}{P_{n} P_{n-1}} \sum_{v=1}^{n-1} \Delta\left(P_{v-1} \lambda_{v}\right) \sum_{r=1}^{v} P_{r} A_{r}(t)+\frac{p_{n}}{P_{n}} \lambda_{n} \sum_{v=1}^{n} P_{v} A_{v}(t) \\
& =O(1)\left\{\frac{p_{n}}{P_{n} P_{n-1}} \sum_{v=1}^{n-1}\left(P_{v} \lambda_{v}-p_{v} \lambda_{v}-P_{v} \lambda_{v+1}\right) P_{v}\right\}+O(1) p_{n} \lambda_{n} \\
& =O(1)\left\{\frac{p_{n}}{P_{n} P_{n-1}} \sum_{v=1}^{n-1} P_{v} P_{v} \lambda_{v}-\frac{p_{n}}{P_{n} P_{n-1}} \sum_{v=1}^{n-1} P_{v} p_{v} \lambda_{v}+p_{n} \lambda_{n}\right\} \\
& =O(1)\left\{T_{n, 1}(t)+T_{n, 2}(t)+T_{n, 3}(t)\right\} .
\end{aligned}
$$

Since

$$
\left|T_{n, 1}(t)+T_{n, 2}(t)+T_{n, 3}(t)\right|^{k} \leq 3^{k}\left\{\left|T_{n, 1}(t)\right|^{k}+\left|T_{n, 2}(t)\right|^{k}+\left|T_{n, 3}(t)\right|^{k}\right\},
$$

to complete the proof of Theorem 3.5, it is sufficient to show that

$$
\sum_{n=1}^{\infty}\left(\frac{P_{n}}{p_{n}}\right)^{k-1}\left|T_{n, r}(t)\right|^{k}<\infty, \text { for } r=1,2,3 .
$$


Now, applying Hölder's inequality with indices $k$ and $k^{\prime}$, where $1 / k+1 / k^{\prime}=1$ and by using (3.7), we get that

$$
\begin{aligned}
\sum_{n=2}^{m+1}\left(\frac{P_{n}}{p_{n}}\right)^{k-1}\left|T_{n, 1}(t)\right|^{k} & \leq \sum_{n=2}^{m+1} \frac{p_{n}}{P_{n} P_{n-1}}\left\{\sum_{v=1}^{n-1} P_{v} P_{v} \Delta \lambda_{v}\right\} \times\left\{\frac{1}{P_{n-1}} \sum_{v=1}^{n-1} P_{v} P_{v} \Delta \lambda_{v}\right\}^{k-1} . \\
& =O(1) \sum_{n=2}^{m+1} \frac{p_{n}}{P_{n} P_{n-1}} \sum_{v=1}^{n-1} P_{v} P_{v} \Delta \lambda_{v} \\
& =O(1) \sum_{v=1}^{m} P_{v} P_{v} \Delta \lambda_{v} \sum_{n=v+1}^{m+1} \frac{p_{n}}{P_{n} P_{n-1}} \\
& =O(1) \sum_{v=1}^{m} P_{v} \Delta \lambda_{v}=O(1) \quad \text { as } m \rightarrow \infty,
\end{aligned}
$$

$$
\begin{aligned}
\sum_{n=2}^{m+1}\left(\frac{P_{n}}{p_{n}}\right)^{k-1}\left|T_{n, 2}(t)\right|^{k} & \leq \sum_{n=2}^{m+1} \frac{p_{n}}{P_{n} P_{n-1}}\left\{\sum_{v=1}^{n-1}\left(P_{v} \lambda_{v}\right)^{k} p_{v}\right\} \times\left\{\frac{1}{P_{n-1}} \sum_{v=1}^{n-1} p_{v}\right\}^{k-1} . \\
& =O(1) \sum_{v=2}^{m+1} \frac{p_{n}}{P_{n} P_{n-1}} \sum_{v=1}^{n-1}\left(P_{v} \lambda_{v}\right)^{k} p_{v} \\
& =O(1) \sum_{v=1}^{m}\left(P_{v} \lambda_{v}\right)^{k} p_{v} \sum_{n=v+1}^{m+1} \frac{p_{n}}{P_{n} P_{n-1}} \\
& =O(1) \sum_{v=1}^{m}\left(P_{v} \lambda_{v}\right)^{k} \frac{p_{v}}{P_{v}} \\
& =O(1) \sum_{v=1}^{m}\left(P_{v} \lambda_{v}\right)^{k-1} p_{v} \lambda_{v} \\
& =O(1) \sum_{v=1}^{m} p_{v} \lambda_{v}=O(1) \quad \text { as } m \rightarrow \infty
\end{aligned}
$$

by virtue of the hypotheses and Lemma 3.2. Finally, as in $T_{n, 1}(t)$, we have that

$$
\begin{aligned}
\sum_{n=1}^{m}\left(\frac{P_{n}}{p_{n}}\right)^{k-1}\left|T_{n, 3}(t)\right|^{k} & =\sum_{n=1}^{m}\left(P_{n} \lambda_{n}\right)^{k-1} p_{n} \lambda_{n} \\
& =O(1) \sum_{n=1}^{m} p_{n} \lambda_{n}=O(1) \quad \text { as } m \rightarrow \infty,
\end{aligned}
$$

Therefore, we get that

$$
\sum_{n=1}^{m}\left(\frac{P_{n}}{p_{n}}\right)^{k-1}\left|T_{n, r}(t)\right|^{k}=O(1) \quad \text { as } m \longrightarrow \infty, \text { for } r=1,2,3 .
$$

This completes the proof of Theorem 3.5.

As a special case the following results can be obtained from Theorem 3.5.

(1) If we take $p_{n}=1$ for all values of $n$, then we get a result concerning the $|C, 1|_{k}$ summability factors of Fourier series. 
(2) If we take $k=1$ and $p_{n}=1 / n$, then we get another new result related to $|R, \log n, 1|$ summability factors of Fourier series.

\section{REFERENCES}

[1] S. Baron, Local property of absolute summability of Fourier series, Tartu Riikl. Ül. Toimetised Vih. 177 (1965), 106-120. MR 35\#2073. Zbl 166.07201.

[2] S. N. Bhatt, An aspect of local property of $|R, \log n, 1|$ summability of Fourier series, Tohoku Math. J. (2) 11 (1959), 13-19. MR 21\#6504. Zbl 107.05303.

[3] _ An aspect of local property of $|R, \log n, 1|$ summability of the factored Fourier series, Proc. Nat. Inst. Sci. India Part A 26 (1960), 69-73. MR 28\#1444. Zbl 098.04401.

[4] H. Bor, On two summability methods, Math. Proc. Cambridge Philos. Soc. 97 (1985), no. 1, 147-149. MR 86d:40004. Zbl 554.40008.

[5] _ Local property of $\left|\bar{N}, p_{n}\right|_{k}$ summability of factored Fourier series, Bull. Inst. Math. Acad. Sinica 17 (1989), no. 2, 165-170. MR 91e:42007. Zbl 697.42006.

[6] On On the local property of $\left|\bar{N}, p_{n}\right|_{k}$ summability of factored Fourier series, J. Math. Anal. Appl. 163 (1992), no. 1, 220-226. MR 93b:42013. Zbl 764.42002.

[7] D. Borwein, The nonlocal nature of the summability of Fourier series by certain absolute Riesz methods, Proc. Amer. Math. Soc. 114 (1992), no. 1, 89-94. MR 92c:42006. Zbl 753.42006 .

[8] G. H. Hardy, Divergent Series, Oxford, at the Clarendon Press, 1949. MR 11,25a. Zbl 032.05801.

[9] M. Izumi and S. Izumi, Localization problem of the absolute Riesz and absolute Nörlund summabilities of Fourier series, Canad. J. Math. 22 (1970), 615-625. MR 41\#8915. Zbl 205.08501.

[10] K. Matsumoto, Local property of the summability $\left|R, \lambda_{n}, 1\right|$, Tohoku Math. J. (2) 8 (1956), 114-124. MR 18,208b. Zbl 071.28403.

[11] S. M. Mazhar, On the summability factors of infinite series, Publ. Math. Debrecen 13 (1966), 229-236. MR 35\#3313. Zbl 166.31602.

[12] K. N. Mishra, Multipliers for $\left|\bar{N}, p_{n}\right|$ summability of Fourier series, Bull. Inst. Math. Acad. Sinica 14 (1986), no. 4, 431-438. MR 88e:42013. Zbl 635.42011.

[13] R. Mohanty, On the summability $|R, \log w, 1|$ of a Fourier series, J. London Math. Soc. 25 (1950), 67-72. MR 11,592b. Zbl 035.33602.

BOR: DEPARTMENT OF MATHEMATICS, ERCIYES UNIVERSITY, 38039 KAYSERI, TURKEY

E-mail address: bor@erciyes.edu.tr 


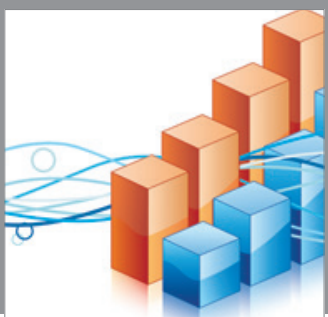

Advances in

Operations Research

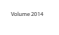

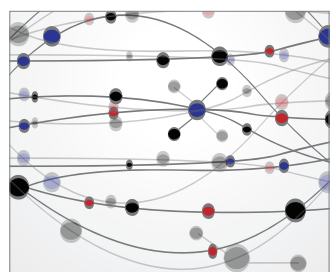

\section{The Scientific} World Journal
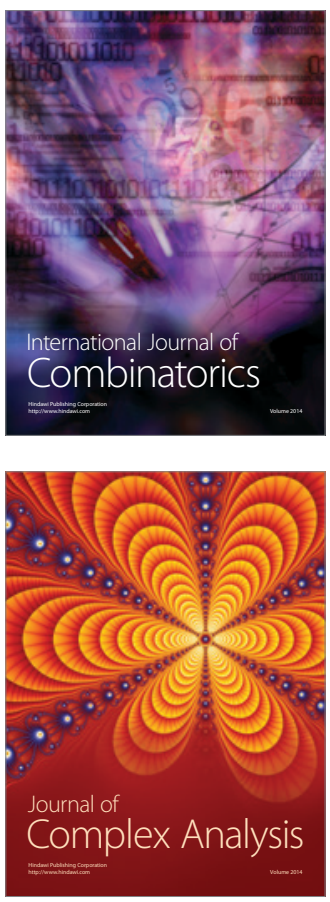

International Journal of

Mathematics and

Mathematical

Sciences
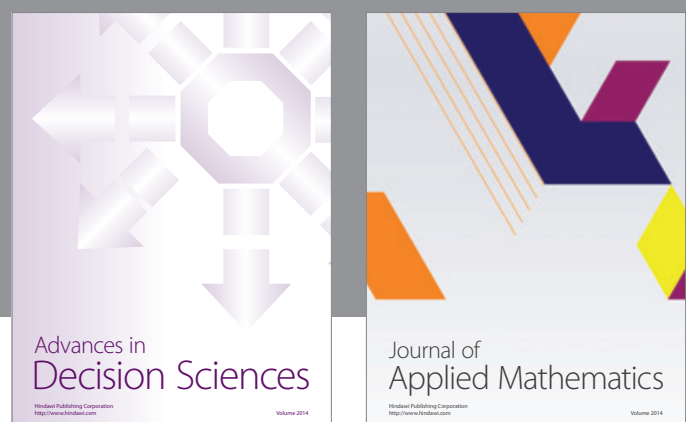

Journal of

Applied Mathematics
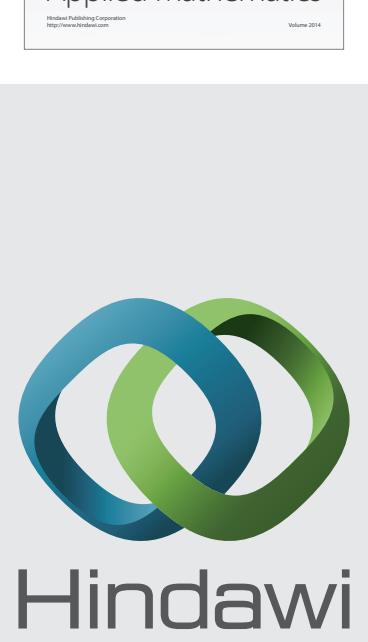

Submit your manuscripts at http://www.hindawi.com
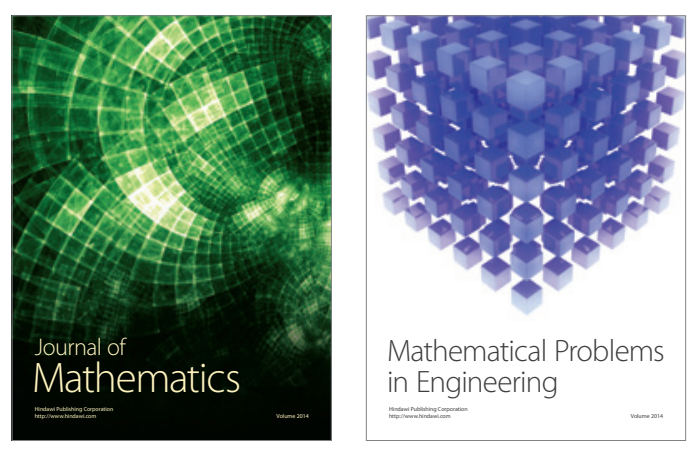

Mathematical Problems in Engineering
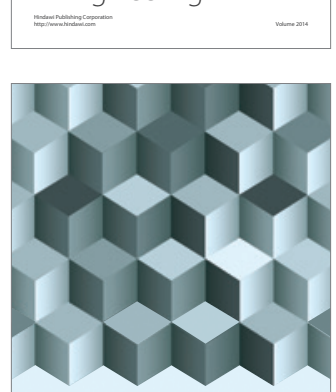

Journal of

Function Spaces
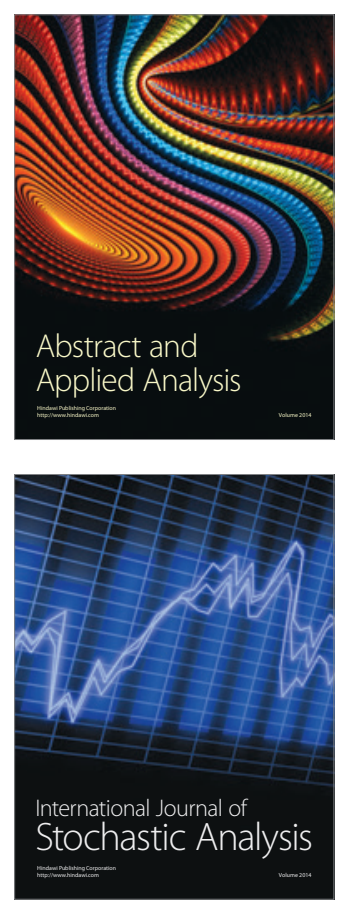

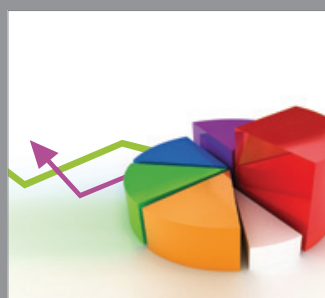

ournal of

Probability and Statistics

Promensencen
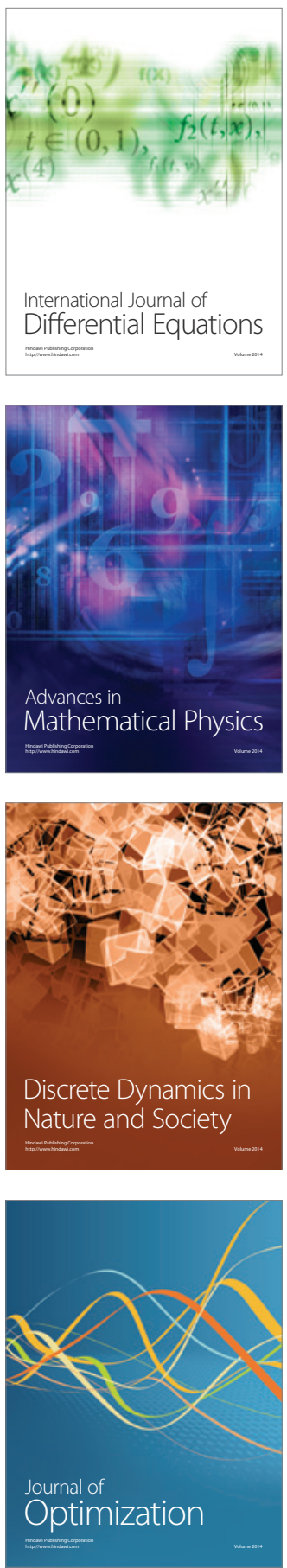